\title{
Fatores que influenciam na escolha pela área cirúrgica em estudantes de Medicina
} Factors that influence the choice of surgical area in medical students

\author{
Izabela Guimarães Augusto ${ }^{1}$ \\ Aline Emanuele Ferreira Oliveira ${ }^{2}$ \\ Phillipe Augusto Marques Silva ${ }^{3}$ \\ Letícia Silva Oliveira $^{4}$
}

\begin{abstract}
RESUMO
Vários médicos mudam suas escolhas de carreira devido a inúmeros fatores influenciadores, porém, muitos também estão inclinados a determinadas especialidades que lhes garantam alto padrão econômico ou prestígio na sociedade independente das exigências. Assim como em outros campos de trabalho, a Medicina, e mais especificamente a cirurgia, passa por um processo elevado de competição, com um número grande de pessoas interessadas a se destacarem no mercado. A atuação na área cirúrgica necessita de muitas qualidades pessoais e profissionais, algumas dessas são adquiridas com treinamento durante a universidade ou algum curso e outras são inerentes à personalidade do indivíduo. Através de metodologia específica, todas as possibilidades de influência de fatores internos e externos na escolha da área cirúrgica, e observado que de forma geral o "ser cirurgião" possui muitas particularidades, a hipótese levantada por esta pesquisa é que: o perfil idealizado do cirurgião aliado a um conjunto de habilidades e competências prévias, relacionadas a personalidade, são fundamentais para a escolha pela área cirúrgica em estudantes de Medicina, enquanto que o objetivo geral é analisar quais são os principais fatores que influenciam os estudantes de Medicina e os profissionais já atuantes a escolherem a carreira cirúrgica. Este estudo qualitativo descritivo e transversal será realizado com estudantes de medicina que possuem interesse na área cirúrgica de três diferentes universidades da região metropolitana de Belo Horizonte através de um questionário estruturado criado pelos autores.
\end{abstract}

Palavras chave: Cirurgiões. Perfil de Cirurgia. Estudantes de Medicina. Residência Médica.

\footnotetext{
Artigo recebido em 03 de outubro de 2019 e aprovado em 17 de janeiro 2020

${ }^{1}$ Graduanda em Medicina pela Pontifícia Universidade Católica de Minas Gerais (PUC Minas), Campus Betim Brasil. E-mail: izabelagaugusto@ hotmail.com

${ }^{2}$ Graduanda em Medicina pela Pontifícia Universidade Católica de Minas Gerais (PUC Minas), Campus Betim Brasil. E-mail: aline.emanuele @ sga.pucminas.br

${ }^{3}$ Graduando em Medicina pela Pontifícia Universidade Católica de Minas Gerais (PUC Minas), Campus Betim Brasil. E-mail: phillipe_augusto@hotmail.com

${ }^{4}$ Graduanda em Medicina pela Pontifícia Universidade Católica de Minas Gerais (PUC Minas), Campus Betim Brasil. E-mail: leticia.oliveiras@live.com
} 


\begin{abstract}
Many doctors change their career choices due to a number of influencing factors, but many are also inclined to certain specialties that guarantee them high economic standards or prestige in society regardless of requirements. As in other fields of work, medicine, and more specifically surgery, goes through a high process of competition, with a large number of interested people standing out in the market. Operating in the surgical field requires many personal and professional qualities, some of which are acquired with training during university or some course and others are inherent to the personality of the individual. Through specific methodology, all the possibilities of influence of internal and external factors in the choice of the surgical area, and observed that in general "being a surgeon" has many particularities, the hypothesis raised by this research is that: the idealized profile of the surgeon Allied to a set of previous personality-related skills and competences are fundamental for the choice of the surgical area in medical students, while the general objective is to analyze what are the main factors that influence medical students and already working professionals. to choose the surgical career. This descriptive and cross-sectional qualitative study will be conducted with medical students who are interested in the surgical area of three different universities in the metropolitan region of Belo Horizonte through a structured questionnaire created by the authors.
\end{abstract}

Key words: Surgeons. Surgery Profile. Medicine Students. Medical Residency.

\title{
INTRODUÇÃO E OBJETIVOS
}

Ao longo da vida as pessoas se envolvem com determinadas áreas de conhecimento, exercitam e se motivam quanto a determinadas habilidades. Este envolvimento e a descobertas de competências podem ser fundamentais para as escolhas profissionais. A decisão da futura profissão pode ser mais ampla do que o simples destaque aos pontos fortes de ordem técnica, pois também está relacionada com a construção a partir das experiências e dos significados que cada pessoa possui.

Neste contexto, a escolha pela carreira médica possui vários significados. São múltiplos os fatores que levam as pessoas a estudar Medicina. Isto pode se dar por motivações de natureza consciente e inconsciente, que vão desde o prestígio social e o saber historicamente existentes, até a atração pela responsabilidade e pelo dinheiro, passando pela necessidade de tornar-se útil, aliviar os que sofrem e modificar a realidade dos demais.

O desejo de estudar Medicina tem se tornado cada vez mais comum. Segundo o Conselho Federal de Medicina (2018), atualmente, existem mais de 270 escolas médicas 
no Brasil, com uma média de 50 alunos por turma regular. Ainda segundo a mesma instituição, 62,5\% dos médicos formados possui pelo menos um título de especialista, revelando a tendência destes novos estudantes optarem por uma formação além da formação generalista do curso. Dentro desse novo universo da especialização o aluno recém-formado novamente se depara com diversos fatores de ordem pessoal e profissional que podem nortear toda a continuidade de sua trajetória.

O exercício profissional do médico é heterogêneo devido ao grande número de especialidades e consequentemente subespecialidades existentes, que se caracterizam por diferenças na área de atuação, no ambiente de trabalho e na variedade dos pacientes. A percepção da área médica de maior afinidade pode ocorrer bem antes da entrada em um curso de Medicina, pelo simples fato de todos os estudantes possuírem um perfil médico baseado no conhecimento empírico da sociedade e na vivência interpessoal e cultural. Certamente é durante o curso de Medicina que os alunos se deparam com a realidade e terão o norteamento quanto à especialidade que seguirão de forma mais efetiva, por meio da orientação dos professores, perfil institucional e das oportunidades construídas. Contudo, muitos mudam suas escolhas devido a inúmeros fatores influenciadores, como a redução do tempo livre para o lazer ou a sobrecarga de horas de trabalho que os impedem de realizar outras atividades recreativas, deixando as necessidades de qualidade de vida pessoal sobressaírem sobre as escolhas profissionais. Entretanto muitos também estão inclinados a determinadas especialidades que lhes garantam alto padrão econômico ou prestígio na sociedade independente das exigências. Assim é possível perceber que a escolha da área médica é um assunto delicado e dependente da análise de muitas variáveis.

Segundo Borges e Savickas (2014) o campo da psicologia a décadas já se dedica a encontrar métodos seguros para prever a escolha da especialidade médica. Assim, inúmeras avaliações de cunho psicológico, como por exemplo as avaliações vocacionais, já tentaram serem submetidas a adaptações a fim de auxiliar na escolha da especialidade médica. Contudo, ainda não foi escolhido nenhum método reconhecido como padrão-ouro para esta finalidade.

O conceito de cirurgião, segundo o Colégio Brasileiro de Cirurgiões (2018), é um profissional médico que executa cirurgias. Também de acordo com esta instituição, cirurgia é uma vasta categoria de tratamentos da medicina invasivos que envolvem a abertura de um corpo, humano ou animal, por uma razão como remover um órgão doente ou para reparar alguma ruptura. O século XIX caracterizou-se pelo 
desenvolvimento do conhecimento que auxiliaram o crescimento da cirurgia e a necessidade do emprego de técnicas especiais proporcionaram o surgimento dos especialistas (COLÉGIO BRASILEIRO DE CIRURGIÕES, 2018). Assim, a cirurgia foi e é uma área de destaque na Medicina e exige cada vez mais profissionais capacitados e comprometidos com as responsabilidades que estas especialidades exigem.

Muitas entidades médicas do Brasil e do mundo se empenham em ordenar as especialidades mais populares, e neste cenário é possível afirmar que as especialidades cirúrgicas possuem uma alta demanda. Para exemplificar, o Conselho Federal de Medicina, em divulgação realizada em 2018, afirmou que a Cirurgia Geral é a terceira especialidade com maior número de especialistas no Brasil e representa aproximadamente $10 \%$ das vagas de residência ofertadas. Fang e Lii (2015) em levantamentos semelhantes afirmaram que as especialidades cirúrgicas ocupam a quinta posição mais popular ao redor do mundo. De forma geral, as especialidades cirúrgicas possuem alta procura e a possibilidade de grandes carreiras.

Assim como em outros campos de trabalho, a Medicina, e mais especificamente a cirurgia, passa por um processo elevado de competição, com um número grande de pessoas interessadas a se destacarem no mercado. Dessa forma, o processo de formação dos estudantes de medicina que se interessam pela área cirúrgica deve se adaptar para selecionar pessoas realmente comprometidas com a prestação de um bom serviço, uma vez que, é inerente o grau elevado de responsabilidade deste futuro profissional (FANG E LII, 2014). Estes mesmos autores destacam que profissionais inseridos em funções que lhes são compatíveis são capazes de oferecer um melhor serviço para seu consumidor final, neste caso, os pacientes.

Até mesmo o mundo televisivo, por meio de inúmeras séries de grande audiência mundial, entendeu que os médicos, e mais especificadamente, os cirurgiões, possuem um estereótipo comportamental e de estilo de vida altamente marcados, o que de certa forma reflete o interesse das pessoas neste perfil profissional (ROTGE, 2015).

A escolha da especialidade é uma importante decisão que define a carreira profissional de um médico. Um aluno de Medicina geralmente faz reflexões ao longo de toda a sua trajetória a respeito de sua personalidade, estilo de vida, valores pessoais e perspectivas de sucesso profissional. Berger et. al. (2017) reforça que os estudos referentes aos fatores que influenciam a escolha dentro do campo da Medicina são predominantemente observacionais e provenientes de outros países da América do 
Norte e Europa, que possuem particularidades no ensino médico. No Brasil, os estudos são escassos.

As especialidades e consequentemente subespecialidades são muito diversas, e de certa forma possuem no imaginário social e na realidade dos processos de trabalho muitos perfis que as caracterizam. Além disso as vivências construídas ao longo do curso são fundamentais para ampliar a visão do futuro médico. Entendendo que as especialidades cirúrgicas possuem algumas especificidades muito marcantes e que o interesse por estas é comprovadamente elevado, entender os significados da opção pela vida de cirurgião desde a graduação é a construção de um conhecimento importante para os estudantes e até mesmo para os médicos cirurgiões que estarão à frente do ensino destes jovens. Foster et. al. (2010) salientam que não existem estudos de preditores que auxiliem na escolha das especialidades médicas.

Logo é relevante conhecer os fatores que influenciam a escolha dos estudantes de Medicina pelas áreas cirúrgicas pois é um desafio e uma necessidade que envolve a adequação e a busca por melhorias das instituições de formação, incluindo escolas médicas e blocos cirúrgicos, professores e preceptores, os alunos e futuros profissionais e principalmente os pacientes e a dinâmica dos serviços de saúde. Trabalhos desta natureza podem ajudar no aconselhamento e assistência dos estudantes, antecipando o planejamento da carreira. Carreiras bem planejadas tem mais chance de sucesso e são importantes para preservar a qualidade de vida de todos os envolvidos no dia a dia da área cirúrgica.

\section{METODOLOGIA}

Este estudo constitui uma revisão bibliográfica a respeito da compressão dos principais fatores que influenciam a escolha da área cirúrgica pelos estudantes de Medicina de diferentes universidades.

A coleta de dados foi realizada no período de 01 a 20 de Janeiro de 2019, e utilizou-se para a pesquisa as bases de dados Literatura Latino-Americana e do Caribe em Ciências da Saúde (LILACS), Scientific Eletrônic Library Online (SCIELO) e National Library of Medicine (PUBMED).

Foi definido como critério de inclusão: artigos publicados entre os anos de 2009 e 2018, pois levantamentos iniciais mostraram que o tema estudado não é muito abordado em pesquisas qualitativas demonstrando a necessidade da pesquisa científica 
deste tema. Outro critério a considerar diz respeito aos Descritores em Ciências da Saúde. Foram incluídos neste estudo artigos que apresentassem descritores como: Cirurgiões, Perfil de Cirurgia, Estudantes de Medicina, Residência Médica.

Após a seleção dos artigos conforme os critérios de inclusão previamente definidos, foram seguidos, nessa ordem: leitura exploratória; leitura seletiva e escolha do material que se adequam aos objetivos e tema deste estudo; leitura analítica e análise dos textos, finalizando com a realização de leitura interpretativa e redação. Após estas etapas, constituiu-se um corpus do estudo agrupando os temas mais abordados nas seguintes categorias: o perfil da área cirúrgica: mito ou realidade, relações da escolha pela área cirúrgica pelos estudantes de Medicina: desafios e reflexões e a escolha da especialidade médica: o perfil pessoal influenciando na carreira profissional.

\section{CONCLUSÕES}

A análise dos fatores que influenciam a escolha do estudante de Medicina pela área cirúrgica depende do entendimento de uma infinidade de variáveis. Dessa forma, a fim de entender o contexto dessa problemática, alguns eixos teóricos da literatura existentes foram estudados.

A abrangência dos eixos escolhidos perpassa desde os conhecimentos já existentes dos motivos que influenciam a escolha profissional, ao perfil da área cirúrgica já existente, que fornecerá a visão já instalada desses profissionais até finalmente as análises dos fatores já apontados por diversos autores que norteiam as escolhas dos estudantes de Medicina pela cirúrgica.

A atuação na área cirúrgica necessita de muitas qualidades pessoais e profissionais. Algumas dessas são adquiridas com treinamento durante a universidade ou algum curso e outras são inerentes à personalidade do indivíduo. De acordo com Greenburg apud Tornetta (2016), alguns programas de treinamento de cirurgiões preferem médicos que exibem uma certa personalidade cirúrgica que vai além do talento em si. A grosso modo, cirurgiões, independente na subárea, optam por funções que gerem uma melhora imediata do paciente e que mostre quase que instantaneamente os resultados (SUBAIT et. al., 2017).

Drosdeck et. al. (2015) afirma que a personalidade cirúrgica é extremamente complexa, mas características inatas podem ser lapidadas após a entrada no meio. $\mathrm{O}$ teste do FFM aplicado por este autor em 150 cirurgiões dos EUA revelou que os 
cirurgiões são predominantemente extrovertidos e muito pouco amáveis. Além disso, as respostas revelam que a maioria possui atenção elevada aos detalhes, forte responsabilidade com compromissos assumidos e desejo forte de perfeição. $\mathrm{O}$ autor também afirmou a existência de uma crença popular que os cirurgiões são pouco amáveis e extremamente confiantes, corroborada em parte, pelos resultados da pesquisa. Preece e Cope (2016) afirmam a existência de uma personalidade cirúrgica influente no sucesso profissional e destacam que o método de aprendizagem mais utilizado por estas pessoas na graduação e residência é o visual, com números bem superiores.

Estas características encontradas elevam muito o nível de estresse e burnout entre esses profissionais, mas os entrevistados consideram o estresse parte inerente do trabalho. Alnassar et. al. (2012) defende que o estresse agudo é reconhecido como fator significativo para o sucesso na sala de cirurgia, pois isto garante que lidem rapidamente com a tomada de decisão na sala cirúrgica e com as adversidades. Assim o fator estresse é percebido diferente entre observadores (restante da equipe) e cirurgiões. Os últimos não entendem que estão sujeitos a níveis elevados de estresse enquanto os primeiros admiram a intensidade elevada que os cirurgiões são submetidos diariamente.

O tempo total de formação também deve ser considerado, uma vez que as áreas cirúrgicas geralmente levam anos para serem concluídas. Isso reflete o grau necessário de especialização e de treinamento destes profissionais, que vai de encontro ao estereótipo de pessoas que passam uma grande parte de suas vidas dentro dos hospitais (ALNASSAR et. al., 2012).

A equipe por sua vez é um desafio na vida do cirurgião. Os conflitos são constantes, pois o cirurgião de forma geral tem que lidar com um misto de comunicação múltipla, ruídos, problemas técnicos, fadiga, problemas institucionais e ansiedade de todos os envolvidos. O status de liderança é recorrente na cultura hospitalar, sendo que estes normalmente atribuem o sucesso nas mãos do cirurgião que raramente nega sua participação mais efetiva (ALNASSAR et. al., 2012).

Os residentes de cirurgia apesar de ainda não estarem a frente da tomada de decisão final nos processos e ainda não possuir autonomia completa na sala cirúrgica são importantes personagens para dizer sobre a existência de um perfil específico da área. Em um estudo realizado por Foster et. al. (2010), no qual foi aplicado um questionário para identificação de traços de personalidade em 27 cirurgiões e 63 residentes, não foram encontradas diferenças significativas entre esses dois grupos e estes ainda apresentaram forte afinidade no que foi considerado uma escala de 
atividades de interesse. Da mesma forma, Hoffman et. al. (2010) encontrou que cirurgiões, residentes de cirurgia e estudantes de Medicina que se interessam pela área cirúrgica possuem características fortes, como autoridade e liderança, em comum.

Os residentes de cirurgia passam por desgaste emocional, psicológico, cognitivo e emocional. Esses profissionais declaram que são altamente exigidos dentro e fora do hospital. Assim, é possível encontrar familiares e amigos que de certa forma são críticos a profissão escolhida por estes jovens e entendem que o compromisso profissional sobrepõe, na maior parte das vezes o compromisso pessoal (FOSTER et. al., 2010).

Outro aspecto, ainda envolvendo os residentes cirúrgicos, diz respeito as crescentes taxas de abandono da residência ainda no primeiro e segundo anos. Estes números podem variar de $17 \%$ a $26 \%$, considerados extremamente elevados pelas entidades de classe. A maioria afirma que não conseguiu conciliar tempo para a própria vida e tempo para a vida de cirurgião. Esse fato corrobora para a crença de que existe no imaginário social, principalmente do estudante de Medicina, um perfil considerado romântico da cirurgia, pensado de forma extremamente amena e que pode acabar em frustação (FOSTER et. al., 2010). Drosdeck et. al. (2015) também se atenta para os altos índices de desistência nas residências cirúrgicas, afirmando que estes se questionam com muito mais criticidade qual o custo da longevidade na carreira.

Segundo Hill et. al. (2014), a "cultura da cirurgia" é real e percebida por profissionais e não-profissionais. Esta pode ser desencorajadora ou encorajadora do papel social destes profissionais na sociedade. De forma geral alguns pontos são recorrentes e devem ser salientados. Primeiramente a ideia de que os cirurgiões não possuem fraquezas nem emoção (associada a proximidade destes com a morte), que são pessoas dispostas ao sacrífico de tempo e energia de forma ilimitada, são extremamente autoconfiantes (confundido muitas vezes com arrogância), são intimidadores (o que pode transparecer agressividade e grosseria), possuem sentimento de autoridade (e consequentemente liderança), preferem uma comunicação direta (relacionada com a franqueza e objetividade), são muito impessoais (facilmente ligada a ideia de pessoas pouco amáveis) e muito competitivos (dispostos a manter o alto desempenho em todos os setores da vida a custa de qualquer preço).

Além de todas as características acima citadas, o perfil cirúrgico está diretamente relacionado a figura do masculino. Hill et. al. (2014) afirma que o ambiente da sala de cirurgia e a figura do cirurgião são fortemente associados a virilidade, e que as mulheres que se arriscam estão expostas a preconceitos e julgamentos. 
Muitos jovens correm todos os dias atrás do sonho de se tornar médico. Além das intenções mais sentimentais como o desejo de salvar vidas e fazer o bem para o próximo, a pessoa que opta por ser médica geralmente possui, pelo menos parcialmente, traços característicos, não necessariamente determinantes de comportamento. Guraya E Almaramhy (2018) destacam que a seleção descuidada de uma futura especialidade compromete a vida do estudante, no sentido de perda de tempo, dinheiro e danos emocionais e do serviço, com qualidade baixa, mostrando a importância singular do debate sobre essa temática.

Segundo Fang e Lii (2015) estudantes de Medicina geralmente observam e analisam os sentimentos dos outros, gostam de novas experiências, desejam ter sucesso profissional, desejam ter alto desempenho em suas habilidades pessoais, não pedem ajuda facilmente, seguem mais seus instintos do que instruções externas, não se preocupam em satisfazer expectativas dos outros e gostam de compartilhar suas vivências. Logo, é possível perceber que já existem alguns traços comumente encontrados que distinguem os futuros médicos.

É importante salientar que qualquer pessoa pode se tornar apta a qualquer função, desde que esteja empenhada em adquirir as competências necessárias. A ideia da construção de um perfil profissional não exclui as pessoas que de certa forma não se encaixam em tal, mas sim, a necessidade de certo norteamento que auxiliará na elaboração destas competências requeridas.

Borges e Savickas (2014) afirmam que a entrada nas escolas de Medicina seleciona pessoas com habilidades cognitivas homogêneas. Contudo após a formatura, as exigências do treinamento prático são heterogêneas e as demandas de trabalha também o serão. Ainda segundo estes autores, existem mais variedades de características e interesses intraespecialidades do que interespecialidades, o que reforça a infinidade de funções que um médico pode optar.

Um inventário psicométrico, que escalona o estilo de personalidade, o estilo de trabalho, o estilo de liderança e o estilo de assumir riscos, aplicado com alunos de terceiro ano em diante de uma escola de Medicina dos EUA mostrou que estes estudantes possuem traços marcantes em algumas dessas escalas (BORGES E SAVICKAS, 2014). Os alunos foram considerados basicamente fortes na condição de trabalhar com pessoas, ajudar os outros e extroversão.

Apesar disso, segundo Fang e Lii (2015) afirmam que a personalidade é mutável por causa do desenvolvimento pessoal, das realizações conquistadas e da interação com 
o meio que está inserido. Logo, a análise da personalidade dos estudantes de Medicina seria mais fidedigna se realizada no mínimo após o quarto ano de curso.

A trajetória acadêmica possui peso elevado na escolha da especialidade. Com base nesta afirmação, Berger et. al. (2017) defende que as escolas de Medicina, consideradas agentes ativos nesta trajetória, devem recrutar estudantes de diferentes configurações. A heterogeneidade de pessoas pode não limitar o número de pessoas envolvidas com cada campo de trabalho. Além disso afirmam que as escolas devem valorizar programas que falam positivamente de todas as especialidades, sem preferências ou direcionamentos, desde os primeiros períodos, oportunizando o contato similar com todas as principais especialidades.

Pensando na trajetória acadêmica cabe lembrar que a Medicina de forma geral sofreu uma mudança forte nos últimos anos com relação as questões de gênero e questões socioeconômicas. Em 1970, menos de 10\% dos ingressantes nas Escolas Médicas dos EUA eram mulheres. Em 2006, esse número passou para mais de 50\% (GURAYA E ALMARAMHY, 2018). Essas mudanças são relevantes no sentido em que necessitam de uma compreensão mais profunda das aspirações dos estudantes, que também foram modificadas, na busca por orientação para escolha da especialidade.

Além das questões acadêmicas, motivos como finanças (com destaque para as dívidas estudantis), histórico escolar, origem demográfica, interesses extracurriculares, atividades de lazer, histórico médico e estilo de vida são apontados por Berger et. al. (2017) como determinantes na escolha pela especialidade médica futura. A cada ano de curso, os fatores podem ficar mais claros. Subait et. al. (2017) também se ateve as questões de gênero, encontrando que estudantes do sexo masculino optam por carreiras na qual se destaca a diversidade de ações e a competitividade, enquanto estudantes do sexo feminino valorizam oportunidades e prestígio social.

Ao mesmo tempo, Guraya e Almaramhy (2018) apontam os fatores socioeconômicos e organizacionais como os destaques de influência.

Segundo Hoffman et. al. (2010) os residentes ingressos nos últimos anos recebem mais críticas do que os residentes de 20 anos atrás. Isso pode significar que os programas de seleção precisam de atualização e podem não estar contemplando o que realmente importa. Este autor sugere que testes de personalidade, comumente utilizados para testes vocacionais, são uma ferramenta complementar importante para esses processos. 
Um exemplo de teste sugerido é o Five Factor Models (FFM). Este é considerado o padrão de ouro na ciência social, e está na vanguarda de testes de personalidade científicos, sendo uma ferramenta sólida, válida e confiável. É baseado em inferência estatística com cinco traços principais: abertura para experiência, consciosidade, extroversão, agradabilidade e neuroticismo, que são compostos por um total de uma subescala com 30 traços de personalidade, que são divididos nestas cinco dimensões principais (ROTGE et. al, 2015).

Rotge et. al. (2015) utilizou do FFM em 1661 residentes e encontrou uma correlação positiva entre as respostas do questionário e a realidade escolhida e experienciada por estes residentes.

Diversos fatores influenciam na tomada de decisão de um estudante de Medicina no momento da escolha de sua especialização. Como demonstrado por Bellodi (2004), existem critérios fundamentais que podem variar desde a preferência por atividades manuais até o gosto pela obtenção de resultados rapidamente. Comparativamente os estudantes que escolheram a prática da Medicina clínica, justificam sua escolha por sua preferência no maior contato com o paciente e pela predileção por atividades intelectuais.

A cirurgia tem passado nos últimos anos por um processo de intensas mudanças, assim como toda a Medicina. O envelhecimento populacional, o uso crescente da tecnologia e o desenvolvimento de técnicas cada vez mais invasivas, alteram o perfil dos serviços que devem ser ofertados e consequentemente da exigência dos profissionais, que devem considerar esse novo olhar para pensar sobre seu futuro e suas relações de trabalho.

A personalidade está relacionada com a forma natural que uma pessoa se comporta perante uma situação enquanto a escolha vocacional está relacionada com os valores que elas acreditam serem corretos para acionar estes comportamentos (TABER et. al., 2011). Tanto a personalidade quanto a escolha vocacional são geralmente anteriores ao ingresso na faculdade. Hill et. al. (2014) ressalta que a exposição a determinadas experiências na condição de paciente e determinados estilos de vida são influencias fortes nas questões profissionais.

Além disso, os anseios pessoais, são extremamente relevantes na decisão dos estudantes. Guraya e Almaramhy (2018) citam que o jovem que busca a cirurgia médica está interessado em possibilidade de inovação, recompensa financeira, treinamento 
constante, prestígio social, desafio técnico, maiores oportunidades e contato limitado com o paciente.

A Cirurgia Geral, na condição de especialidade cirúrgica mais procurada pelos estudantes, foi citada em estudo desenvolvido por Guraya e Almaramhy (2018) como uma área que aceita pessoas que são ansiosas, céticas, impulsivas, agressivas, hostis e pouco sociáveis. Os estudantes são conscientes destas características, e se identificam com a possibilidade de serem desta forma, sem culpa, em pelo menos uma delas. Estes mesmos estudantes concordam que o potencial prévio é influente nos resultados.

Dentre os fatores que mais pesam para os estudantes, o mentor, o estilo de vida, as horas de trabalho, a remuneração e a família são os mais citados (SCHMIDT et. al., 2016). Da mesma forma, Marshall et. al. (2014) aponta como fatores negativos o estresse, a duração da formação, as horas trabalhadas e a sociabilidade, e como fatores positivos o estilo de vida percebido.

Schmidt et. al. (2016) menciona que nos EUA, estudantes de Medicina consideram a área cirúrgica como um ambiente hostil. Para driblar tal problema, estudos que relacionam a influência positiva de um mentor-cirurgião foram desenvolvidos no Reino Unido. Nestes foi possível estabelecer que os estudantes passam a maior parte da graduação em contato apenas com a Medicina clínica, e que o treinamento tardio é estressante.

Estudantes avaliados após estágio cirúrgico, em estudo desenvolvido por Marshall et. al. (2014), se sentiram mais preparados para fazerem sua escolha por tal área, alegando que o contato com os mentores e com o bloco cirúrgico influencia positivamente desde que aconteçam oportunidades educacionais ativas. No mesmo estudo, os preceptores preferem alunos que se auto intitulam cirurgiões natos. Aqueles que se distinguem na carreira demonstram traços similares aos seus médicos preceptores, que quase universalmente incluem ser bem disciplinado, motivado e voluntariamente admitir seus erros quando necessário (GREENBURG apud TORNETTA, 2016).

Já segundo Berger et. al. (2017) os principais fatores percebidos pelos estudantes se relacionam com potencial salário, carreira do parceiro, estímulo intelectual, horas de treinamento, parentes próximos na mesma carreira, dívidas estudantis, possibilidade de matrimônio e filhos, experiências práticas e principalmente um mentor com escuta ativa e compreensivo. 
Apesar dos trabalhos desenvolvidos no campo da predição das especialidades médicas não terem objetivos claramente deterministas, alguns autores, como Hill et. al. (2014) afirma que quem não se "encaixa” deverá buscar outros pontos de destaque para fazer a diferença no meio. Esta afirmação pode mostrar que muitas pessoas com traços de personalidade diversos são subrepresentadas, como por exemplo as próprias mulheres, mostrando a necessidade de reinterpretação e alargamento da base científica da visão do médico cirurgião.

\section{REFERÊNCIAS}

ALNASSAR SAMI, Hajjar et al. Real-time observations of streesful events in the operating room. Saudi Jornal of Anaesthesia, Riade, v. 6, n. 2, p. 136-139, jun. 2012.

BELLODI, Patrícia Lacerda. Surgery or general medicine: a study of the reasons underlying the choice of medical specialty. Sao Paulo Med. J., São Paulo, v. 122, n. 3, p. 81-86, Maio 2014.

BERGER, Anthony et al. Choosing surgery as a career: early results of a longitudinal study of medical students. Surgery, Iowa, v. 161, n. 6, p. 1683-1689, jun. 2017.

BORGES, Nicole; SAVICKAS, Suzanne. Work style preferences among medical specialties. Journal of Vocational Behavior, Dayton, v. 84, p. 303-306, 2014.

CAVALCANTE, Ricardo Bezerra; CALIXTO, Pedro; PINHEIRO, Marta Macedo Kerr. Análise de conteúdo: considerações gerais, relações com a pergunta de pesquisa, possibilidades e limitações do método. Inf. \& Soc. Est, João Pessoa, v. 24, n. 1, p.1318, abr. 2014.

COLÉGIO BRASILEIRO DE CIRURGIÕES. A evolução da cirurgia. Brasília: CBC, 2018.

CONSELHO FEDERAL DE MEDICINA (BRASIL). Revista de Humanidades Médicas. Brasília: CFM, 2018.

DROSDECK, Joseph et al. Surgeon and nonsurgeon personalities at different career points. Journal of Surgical Research, Columbus, v. 196, p. 60-66, 2015.

FANG, Ji-Tseng; LII, Shu-Chung. Relationship between personality traits and choosing a medical specialties. Journal of the Formosan Medical Association, Taoyuan, v. 114, p. 1116-1121, 2015.

FOSTER, Kevin et al. A Psychological Profile of Surgeons and Surgical Residents. Journal of Surgical Education, Phoenix, v. 67, n. 6, p. 359-370, 2010. 
GURAYA, Salman; ALMARAMHY, Hamdi. Mapping the factors that influence the career specialty preferences by the undergraduate medical students. Saudi Journal of Biological Sciences, Almadinah Almunawwarah, v. 25, p. 1096-1101, 2018.

HILL, Elspeth et al. Can I cut it? Medical student's perpections of surgeons and surgical careers. The American Journal of Surgery, Maastrich, v. 208, p. 860-867, 2014.

HOFFMAN, Bensom et al. Personality differences between surgery residents, nonsurgerys residents, and medical students. Journal of Surgical Education, Durham, v. 148, n. 2, p. 187-193, 2010.

MARSHALL, Dominic et al. Medical student experience in surgery influences their career choices: a systematic review of the literature. Journal of Surgical Education, London, v. 72, n. 3, p. 438-445, jun. 2015.

PREECE, Ryan; COPE, Alexandra. Are surgeons born or made? A comparison of personality traits and learningstyles between surgical trainees and medical students. Journal of Surgical Education, Austin, v. 73, n. 5, p. 768-773, 2016.

ROTGE, Jean-Yves et al. Relationship between personality dimensions and medical specialty in 1661 residents. Journal of Psychosomatic Research, Paris, v. 79, p. 331332, jun. 2015.

SCHMIDT, Lauren et al. Factors influencing US medical students' decision to pursue surgery. Journal of Surgical Research, Buffalo, v. 203, p. 64-74, jun. 2016.

SUBAIT, Abeer Al. Factors influencing the career choices among medical university students of King Saub bin Abdulaziz University, Riyaadh Saudi Arabia; A crosssectional study Design. The Saudi Journal for Dental Research. Riyadh, v. 8, p. 7378, 2017.

TABER, Brian et al. Personality and values as predictors of medical specialty choice. Journal of Vocations Behavior, v. 78, p. 202-209, 2011.

TORNETTA, Paul; BOGDAN, Yelena. CORR ® Curriculum - Orthopaedic Education: Mentorship in Surgical Training. Clinical Orthopaedics And Related Research®, [s.1.], v. 474, n. 5, p.1125-1128, 18 fev. 2016. 